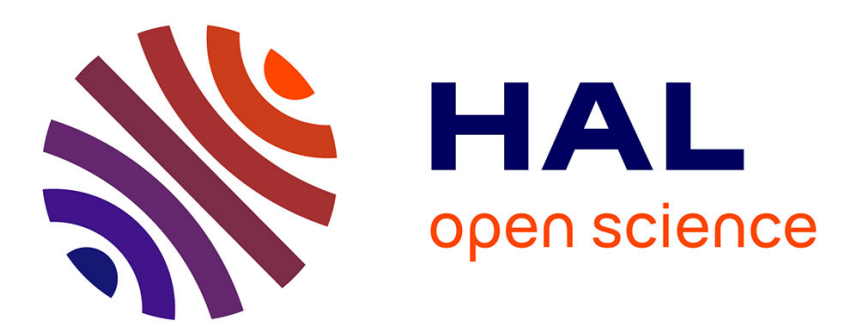

\title{
Multimodal Damping of a Plate With a Passive Piezoelectric Network
}

Boris Lossouarn, Mathieu Aucejo, Jean-François Deü, Kenneth A. Cunefare

\section{To cite this version:}

Boris Lossouarn, Mathieu Aucejo, Jean-François Deü, Kenneth A. Cunefare. Multimodal Damping of a Plate With a Passive Piezoelectric Network. 34th IMAC Conference and Exposition on Structural Dynamics, IMAC XXXIV, Jan 2016, Orlando, United States. hal-01739670

\section{HAL Id: hal-01739670 \\ https://hal.science/hal-01739670}

Submitted on 21 Mar 2018

HAL is a multi-disciplinary open access archive for the deposit and dissemination of scientific research documents, whether they are published or not. The documents may come from teaching and research institutions in France or abroad, or from public or private research centers.
L'archive ouverte pluridisciplinaire HAL, est destinée au dépôt et à la diffusion de documents scientifiques de niveau recherche, publiés ou non, émanant des établissements d'enseignement et de recherche français ou étrangers, des laboratoires publics ou privés. 


\title{
Multimodal Damping of a Plate With a Passive Piezoelectric Network
}

\author{
B. Lossouarn, PhD Student - M. Aucejo, Assistant Professor - J.-F. Deü, Professor \\ Structural Mechanics and Coupled Systems Laboratory, Cnam \\ 2 Rue Conté, 75003 Paris, France \\ K. A. Cunefare, Professor \\ Woodruff School of Mechanical Engineering, Georgia Institute of Technology \\ 813 Ferst Dr, Atlanta, GA 30332, USA
}

\begin{abstract}
Multimodal vibration reduction can be obtained by coupling a mechanical structure to an electrical network approximating its modal properties. This strategy is applied to the control of a clamped plate with a 2D network of passive electrical components. The plate and the network are coupled through a periodic array of piezoelectric patches that enables the energy conversion. The network is experimentally implemented with inductors and transformers in order to create electrical resonances that present a spatial distribution approaching the first mode shapes of the plate. By tuning electrical resonances to mechanical ones, it becomes possible to introduce the equivalent of a tuned mass effect. This effect only occurs if the electrical and mechanical mode shapes are sufficiently close, which requires specific network topology and boundary conditions. Once the network is tuned, adding resistors introduces damping that can lead to a broadband vibration reduction of the plate. It is thus shown that a 2D control can be implemented with a purely passive solution, which could be of great interest for many embedded applications.
\end{abstract}

Keywords: Multimodal damping, Piezoelectric coupling, Periodic array, Resonant network, Passive control

\section{Introduction}

Arrays of piezoelectric patches enables the implementation of efficient control strategies focusing on large wavelength compared to the dimension of the patches. Indeed, using multiple patches of reduced dimensions limits the charge cancellation that would happen with longer patches, which allows a control over a wider frequency range. Several solutions involving an array of piezoelectric shunts have been proposed to damp vibration of plates. Resonant shunts [1, 2] exhibit performance around the electrical resonance but a multimodal control would require the addition of several electrical resonances for each shunt, which would lead to an impractical number of components. Broadband wave attenuation can be obtained with negative capacitance circuits [3] but this solution requires an external power supply. So, the passive implementation of a robust multimodal control of a plate is still an open problem.

A theoretical concept was proposed by Vidoli and dell'Isola [4], who presented an interconnection of piezoelectric patches through a 2D electrical network. An efficient energy transfer is obtained by tuning analogous plate-like electrical modes to the corresponding mechanical modes of the plate. The coupling of two structures exhibiting similar resonances acts as a tuned mass damping of several modes simultaneously. A suitable topology of the electrical network can be determined from the electrical analogue of a plate. Alessandroni et al. [5] used a Lagrangian formulation to find an electrical network as a discrete analogue of a plate unit cell. Unfortunately, the large number of electrical components required for each unit cell of the network makes its practical implementation difficult.

In the present contribution, we focus on a new electrical topology that reduces the number of required components. The proposed electrical network is obtained by extending a procedure previously applied to rods and beams [6, $7,8,9]$. It consists of a finite difference formulation of the equations describing the mechanical medium, followed by a direct electromechanical analogy. A 2D electrical topology involving capacitors, inductors and transformers is thus defined. This network is implemented with passive components in a case focusing on the analogue of a 
clamped plate. The electrical modes are observed experimentally, which validates the analogous electrical topology. Afterward, the control of a clamped plate is considered by a coupling to the electrical network through an array of piezoelectric patches. A significant vibration reduction is observed over a broad frequency range. Depending on the application, the electrical network is then tuned by adding resistors or by modifying the values of the inductors. In the end, a passive and tunable solution for the control of a plate is proposed and experimentally validated.

\section{Electrical analogue of a plate}

An electrical analogue of a plate is found from a discretization of the mechanical medium followed by a direct electromechanical analogy. The resulting electrical network is implemented with passive components and the topology is validated by observing electric modal properties that are analogous to what can be obtained for a clamped plate.

\subsection{Direct electromechanical analogy}

As a two-dimensional extension of the Euler-Bernoulli beam theory, the Kirchhoff-Love plate theory gives a relation between the spatial and temporal derivatives of the transverse displacement $w$ through the equation

$$
D\left(\frac{\partial^{4} w}{\partial x^{4}}+2 \frac{\partial^{4} w}{\partial x^{2} \partial y^{2}}+\frac{\partial^{4} w}{\partial y^{4}}\right)=-\rho h \frac{\partial^{2} w}{\partial t^{2}}, \quad \text { where } \quad D=\frac{Y h^{3}}{12\left(1-\nu^{2}\right)} .
$$

$Y, \nu, \rho$ and $h$ are respectively the Young's modulus, the Poisson's ratio, the density and the thickness of the plate.

$\frac{\partial[.]}{\partial x}$ and $\frac{\partial[.]}{\partial y}$ represent the partial derivative along the principal directions $x$ and $y$. It can be shown that a finite difference procedure applied to those differential operators in Eq. 1 leads to a set of discrete equations that can be represented under the electrical scheme in Fig. 1.

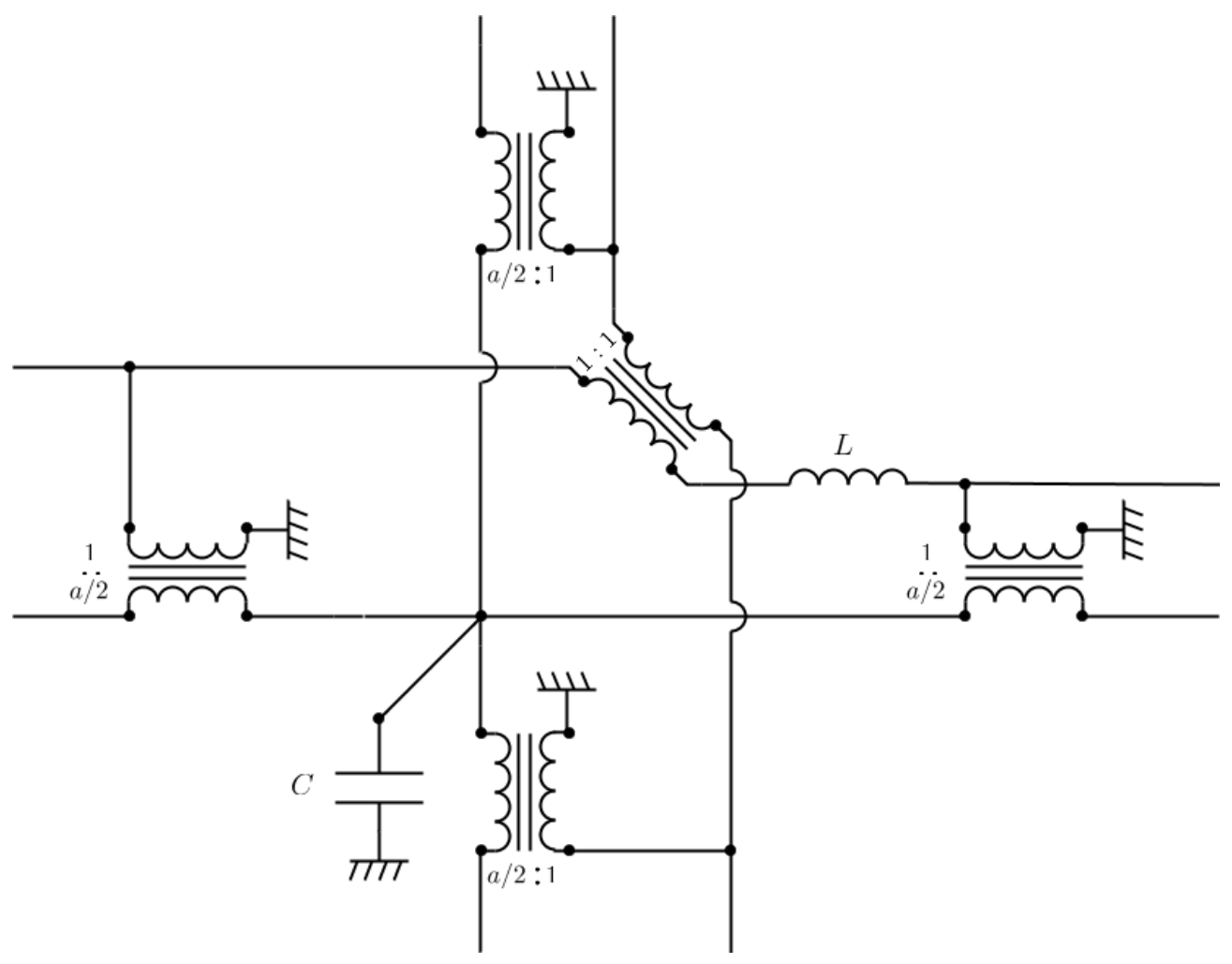

Fig. 1 Electrical unit cell corresponding to the analogue of a discretized square plate

The proposed topology is based on the direct electromechanical analogy, which assumes an equivalence between mass and inductance and between stiffness and the inverse of a capacitance. The inductance $L$ is then equal to $\rho h a^{2}$, which corresponds to the mass of a square unit cell of side $a$. $C$ is equal to $1 / D$, the inverse of the bending 
stiffness of the square plate element. The analogous electrical unit cell can thus be implemented with only one inductor, one capacitor and five transformers.

Note that the connection of adjacent unit cells reduces the number of components. Indeed, a series of two transformers of ratio $a / 2$ can be replaced by a single transformer with a ratio equal to $a$. This simplification does not apply to the boundaries, but the average number of transformers per unit cell still tends to 3 when the number of unit cells is increasing. A simpler network is finally obtained compared to the topology proposed by Alessandroni et al. [5], which involves three inductors, one capacitor and six transformers per unit cell. As a consequence, the new topology simplifies the practical implementation of the analogous network for experimental validation and application to piezoelectric damping.

\subsection{Experimental validation}

In order to validate the electrical topology, we focus on the analogue of a clamped plate discretized with 20 unit cells. The clamped boundary conditions require zero displacement and zero rotation along the 4 edges of the plate. The direct analogue in the electrical domain corresponds to zero current flowing through the edges of the electrical network. Those electrical boundary conditions simplify the network to an electrical analogue made of 6 inductors, 16 capacitors and 23 transformers.

As the proposed electrical network represents the analogue of a clamped plate, it was decided to validate the electrical behavior with classical instrumentation normally dedicated to mechanical modal analysis. The setup is represented in Fig. 2 where a clamped plate and its electrical analogue appear together in order to show the analogy in term of data acquisition. If the considered structure is the mechanical plate, we acquire the input force and the velocity, which is scanned on several points with a vibrometer. If the electrical network is analyzed, the signals of interest are not force and velocity but their direct analogues: an input voltage and the current flowing through the inductors. The direct measurement of the current would require the introduction of instruments in the network, but this could modify its electrical properties. A less intrusive solution consists in measuring the voltage drop across the

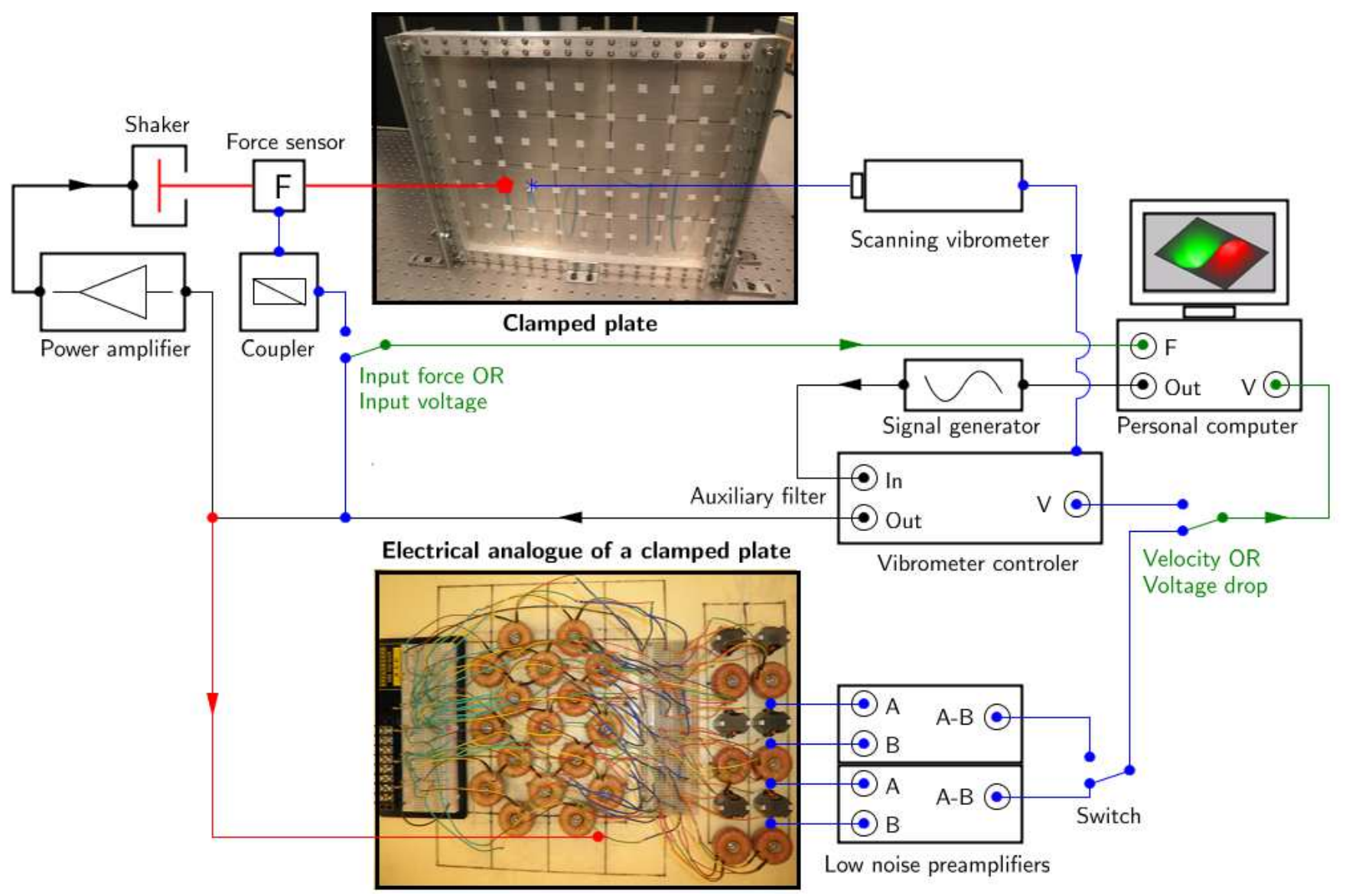

Fig. 2 Experimental setup for the modal analysis of a clamped plate or for its analogous electrical network 


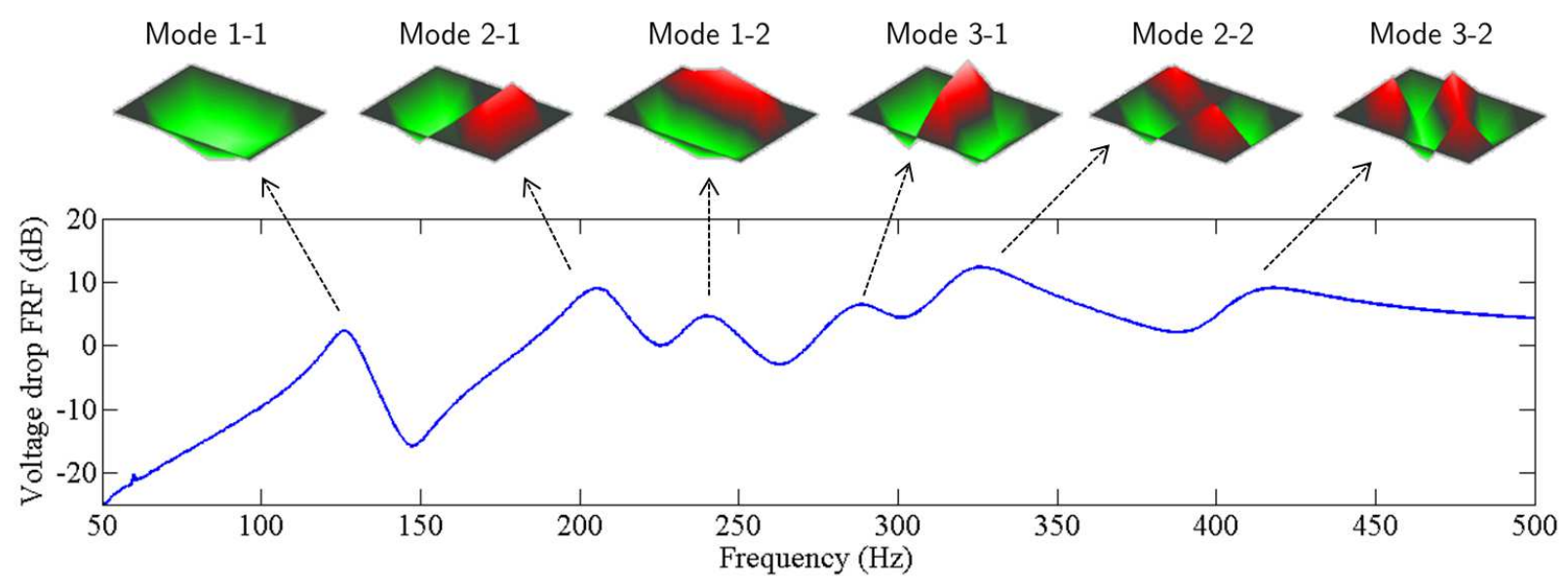

Fig. 3 Electrical frequency response function of the network and the corresponding electrical mode shapes

inductors, which is finally the analogue of the acceleration. This differential voltage is measured by using a low noise preamplifier offering a differential function. The resulting signal and the voltage input are sent to the workstation, where the electrical frequency response function (FRF) is computed.

Electrical mode shapes can be determined by scanning several points of the electrical network. This requires the use of two preamplifiers and a switch, which allows changing the measurement location without any delay. While the voltage drop is measured on one inductor, we have time to prepare the electrical connections for the next measurement. This is crucial, as the scanning process is controlled by the vibrometer software that generates an instantaneous switching between measurement points.

The electrical mode shapes are represented in Fig. 3 together with an electrical FRF measured at locations that are analogous to the points of excitation and measurement represented in Fig. 2 for the clamped plate. The presence of 6 inductors in the network naturally yields 6 electrical resonances. Compared to classical FRFs obtained for a plate, each resonance exhibits a low quality factor, which is due to the internal damping in the electrical components. The spatial distributions of the electrical current are similar to the first 6 mode shapes of a clamped plate. This validates the proposed network topology that comes from the Kirchhoff-Love plate theory.

\section{Multimodal piezoelectric damping}

Once the design of the electrical network is validated, it can be applied to the damping of a clamped plate. The network is coupled to the plate with an array of piezoelectric patches. When electrical modes match their mechanical analogues, significant multimodal vibration reduction is observed.

\subsection{Clamped plate}

The mechanical structure to control is a clamped aluminum plate $400 \mathrm{~mm}$ long, $320 \mathrm{~mm}$ wide and $1.9 \mathrm{~mm}$ thick. The clamping frame is made of square aluminum bars reinforced with steel angle channels. Two rows of bolts are equally tightened with a torque wrench to ensure zero deflection and zero slope boundary conditions. A white noise excitation is generated from a shaker and the transverse velocity of the plate is measured with a laser vibrometer, as represented in Fig. 2. The input force is measured with a force sensor placed between the shaker and the plate. The force signal is then processed together with the velocity signal to compute the velocity FRF.

As seen in Fig. 4, the aluminum plate is covered with an array of 20 square piezoelectric patches of side 72.4 $\mathrm{mm}$ and $0.27 \mathrm{~mm}$ thickness. The patches are made of PZT-5H, which is a soft piezoceramic. As a reference, it is observed that the first mode of the plate is around $140 \mathrm{~Hz}$ with open-circuited patches and this frequency is decreased by $3.5 \%$ once the patches are short-circuited. The frequency range of the analysis was defined according to the number of plate-like modes that can be generated with the electrical network to influence the mechanical frequency responses. We thus focus on the first six modes of the plate, which spread until $600 \mathrm{~Hz}$. The open-circuited FRF is presented in Fig. 5, where six resonances are observed. The distinction between the fourth and fifth resonances is not straightforward because they occur at almost the same frequency. 


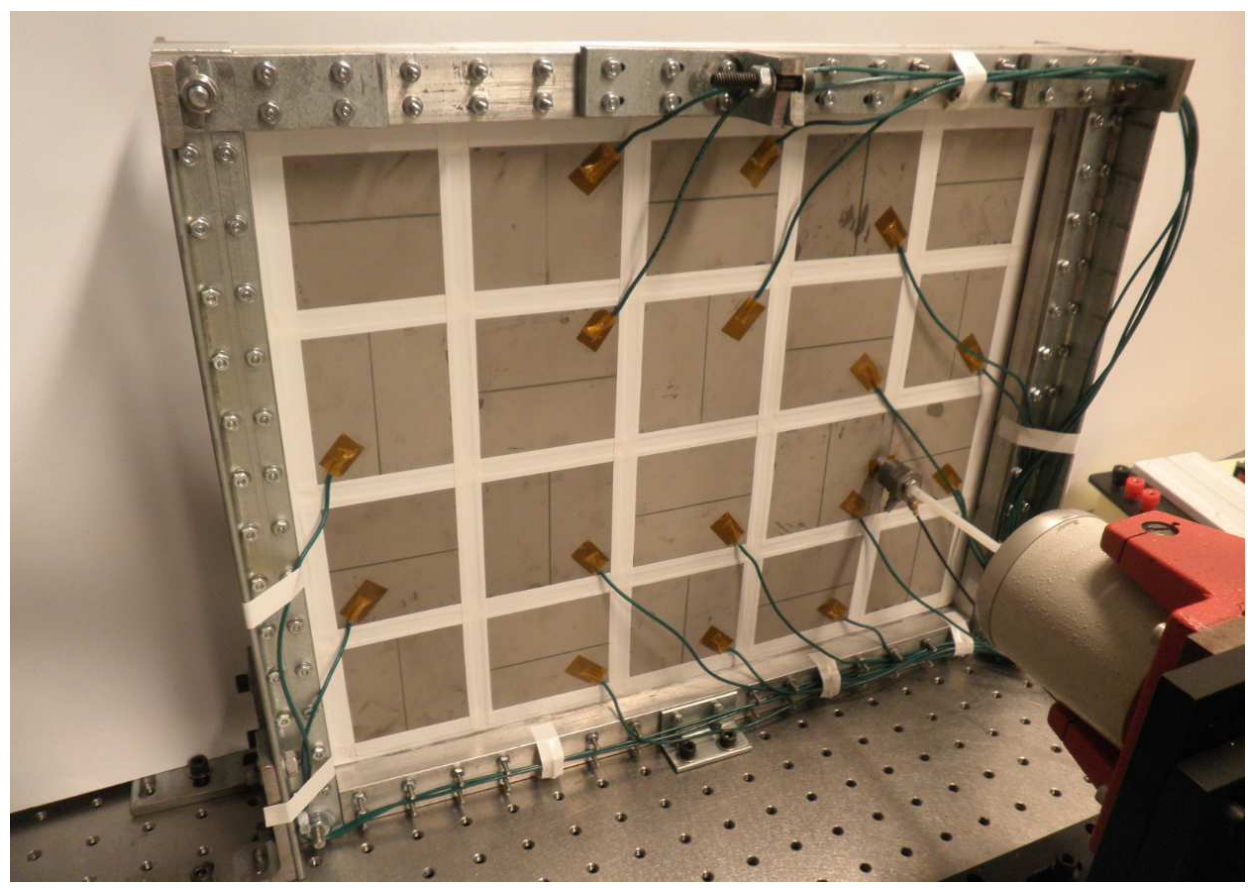

Fig. 4 Clamped aluminum plate covered with 20 square piezoelectric patches

\subsection{Coupling to the electrical network}

The electrical network is connected to the plate by replacing the capacitance $C$, represented in Fig. 1, by the piezoelectric capacitance offered by the patches. Consequently, no external capacitors are required. According to the discrete network topology obtained for a clamped plate, the patches on the corners do not play any role. Only 16 of the 20 patches are thus connected to the electrical network for the present application. Once the network is engaged, it is seen in Fig. 5 that the coupling provides a strong modification of the modal behavior of the plate.

For the measurements presented in Fig. 5, the value of the 6 inductors is equal to $1 \mathrm{H}$, which leads to a suitable tuning of the network around the first mode of the plate. Indeed, we can observe a local minimum around $140 \mathrm{~Hz}$ surrounded by two local maximum that present approximately the same amplitude. This is a characteristic of an underdamped tuned mass control, which is here generated by the resonant network. It is remarked that the vibration reduction is also significant for the higher modes. However, the network does not offer a tuning that is optimized on all the modes simultaneously. This is due to the fact that the electrical network is a discrete structure that only approximates the continuous plate behavior. The lower electrical resonances occur at frequencies that are close to the corresponding mechanical resonances but for higher modes the frequency error becomes non-negligible. It is possible to more closely match the first 6 resonances of the plate with the same network topology by increasing the number of unit cells.

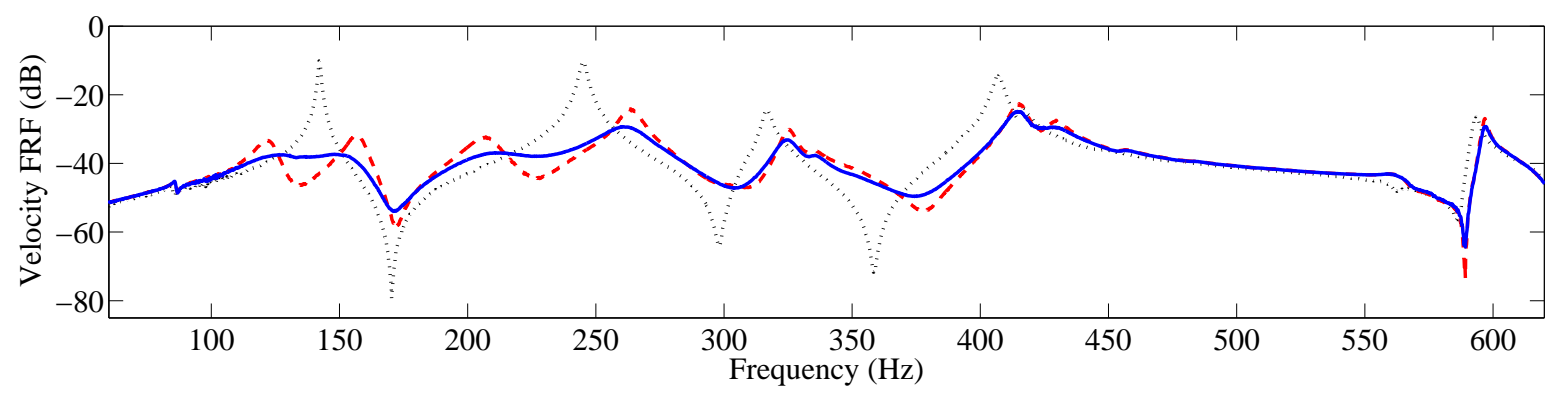

Fig. 5 Effect of the multimodal control on the velocity frequency response function of the plate $-(\cdots)$ with opencircuited patches, $(--)$ with coupling to the analogous electrical network and no external resistance - (-) with coupling to the analogous electrical network and $180 \Omega$ in series with each inductor 


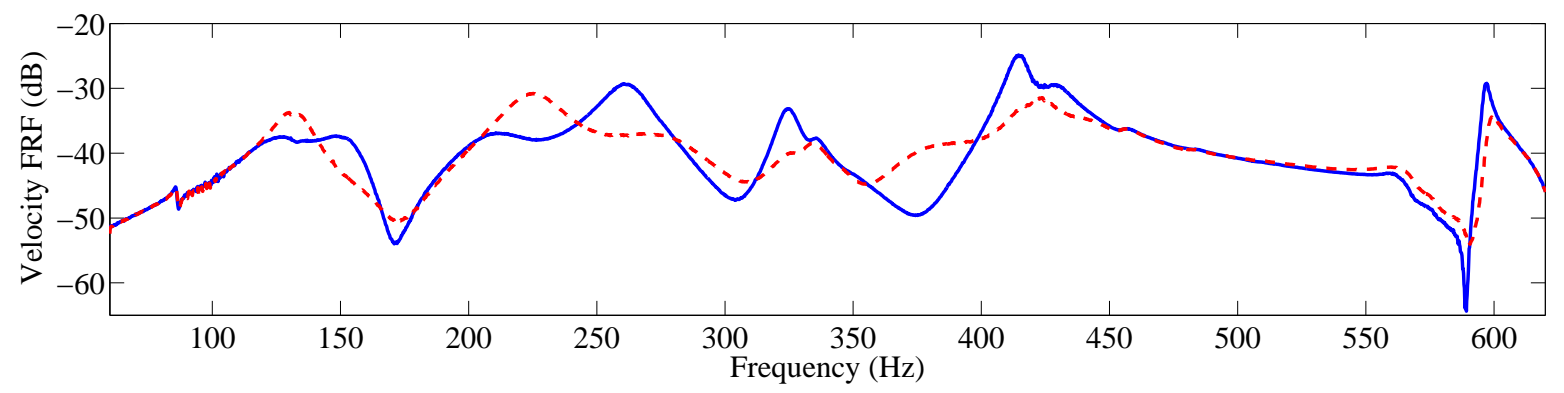

Fig. 6 Tuning of the network - $(-)$ minimization of the amplitude around the first mode with $L=1 \mathrm{H},(--)$ optimization over a broader frequency range with $L=0.8 \mathrm{H}$

\subsection{Tuning procedures}

The network can be tuned in different ways depending on the considered application. For example, if a control is required at one specific frequency which is near a mechanical resonance, the inductors can be adjusted to move the corresponding electrical resonance closer. This would induce an antiresonance, as seen in Fig. 5 around the first and second modes. Then, the internal damping in the components should be minimized in order to increase the depth of the antiresonance.

On the other hand, a control on a broader frequency range requires the introduction of additional damping in the network. Indeed, it is shown in Fig. 5 that $180 \Omega$ resistors in series with the 6 inductors flatten the frequency response function. A vibration reduction close to $27 \mathrm{~dB}$ is then obtained around the first mode. It is noticed that the addition of resistance does not necessarily increase the number of components as it can be included into the design of the inductors. It is seen in Fig. 5 that the introduction of resistors mainly affects the first two modes because of the discretization of the electrical network that yields inaccurate tuning at higher frequencies. However, a solution for a broadband vibration reduction with the considered discrete network can be to detune the first modes to better tune higher modes. This strategy is presented in Fig. 6 , where the previous case involving a network with $1 \mathrm{H}$ inductors and $180 \Omega$ resistors is compared to a second case with $0.8 \mathrm{H}$ inductors and the same resistors. By decreasing the inductance, the electrical resonances move to higher frequencies. In this case, it can be noticed that the second electrical resonance is moved from a frequency below the second mechanical resonance to a frequency above it. Even if the modification of the tuning is not beneficial for the first mode, the maximum of the amplitude over the frequency range of interest is minimized. The detuning of the first mode moves the higher electrical resonances closer to their mechanical analogues, which clearly reduces the amplitude of the frequency response function around the higher modes. It is thus finally shown that the proposed passive damping can induce substantial vibration reduction over a broad frequency range.

\section{Conclusions}

A new electrical topology that approximates the modal properties of a plate was obtained from a finite difference procedure applied to the Kirchhoff-Love equation, followed by a direct electromechanical analogy. The resulting network was implemented with passive components and validated experimentally through a modal analysis procedure similar to what is normally performed for a mechanical structure. Plate-like mode shapes are observed when looking at the distribution of the current over the electrical network. To the best knowledge of the authors, this work presents the first practical implementation of a plate electrical analogue based on the direct electromechanical analogy. It is thus also the first time that such a passive network is connected to a plate for a piezoelectric damping purpose. It is observed that the coupling induces a significant modification of the mechanical response. Depending on the application, a suitable tuning of the electrical network can then optimize the vibration reduction around one specific frequency or over a broad frequency range.

\section{Acknowledgments}

The authors would like to thank the French Ministry of National Education, Higher Education and Research for providing a three year scholarship for doctoral studies related to piezoelectric damping. The authors also sincerely acknowledge the Fulbright Program, which made possible a nine-month visit at the Georgia Institute of Technology. 


\section{References}

[1] Casadei, F., Ruzzene, M., Dozio L. and Cunefare, K. A., "Broadband vibration control through periodic arrays of resonant shunts: experimental investigation on plates," Smart materials and structures, 19(1), p. 015002, 2010.

[2] Chen, S. Wang, G., Wen, J. and Wen, X., "Wave propagation and attenuation in plates with periodic arrays of shunted piezo-patches," Journal of Sound and Vibration, 332(6), pp. 1520-1532, 2013.

[3] Tateo, F., Collet, M., Ouisse M. and Cunefare, K. A., "Design variables for optimizing adaptive metacomposite made of shunted piezoelectric patches distribution," Journal of Vibration and Control, in press, doi:10.1177/1077546314545100, 2014.

[4] Vidoli, S. and Dell'Isola, F., "Vibration control in plates by uniformly distributed PZT actuators interconnected via electric networks," European Journal of Mechanics-A/Solids, 20(3), pp. 435-456, 2001.

[5] Alessandroni, S., Andreaus, U., Dell'Isola, F. and Porfiri, M., "A passive electric controller for multimodal vibrations of thin plates," Computers and structures, 83(15), pp. 1236-1250, 2005.

[6] Lossouarn, B., Aucejo, M. and Deü, J.-F., "Multimodal coupling of periodic lattices and application to rod vibration damping with a piezoelectric network," Smart Materials and Structures, 24(4), p. 045018, 2015.

[7] Lossouarn, B., Aucejo, M. and Deü, J.-F., "Multimodal vibration damping through a periodic array of piezoelectric patches connected to a passive network," Proceedings of SPIE 9431, pp. 94311A-94311A-14, 2015.

[8] Porfiri, M., Dell'Isola, F., and Mascioli, F. M. F., "Circuit analog of a beam and its application to multimodal vibration damping, using piezoelectric transducers," International Journal of Circuit Theory and Applications, 32(4), pp. 167-198, 2004.

[9] Lossouarn, B., Deü, J.-F. and Aucejo, M., "Multimodal vibration damping of a beam with a periodic array of piezoelectric patches connected to a passive electrical network," Smart Materials and Structures, 24(11), p. 115037, 2015. 